\title{
L'IMPORTANZA DELLE CITAZIONI NEL PENDOLO DI FOUCAULT DI UMBERTO ECO E LA LORO TRADUZIONE
}

Parole chiave: Umberto Eco, Pendolo di Foucault, citazione/intertestualità, traduzione.

Le pagine che presentiamo qui non possono che cominciare con le parole del professor Eco, scrittore di un successo che è pari alla sua cultura, ma anche teorico della letteratura tra i più importanti e autorevoli: «Come autore di romanzi che sugli echi intertestuali giocano moltissimo sono sempre stato lieto quando il lettore ha colto il rinvio, la strizzata d'occhio». ${ }^{1}$ Questa citazione rappresenta solo una breve sintesi del pensiero di Eco riguardante il rapporto autore-lettore. È noto che fin dall'inizio della sua carriera egli abbia capito che i legami che si creano tra queste due importanti categorie sono cruciali. Con gli anni, nei suoi libri, sono apparse tutte le fondamentali categorie del suo pensiero - opera aperta, intentio operis, intenzione autoriale, intertestualità, interpretazione, lettore modello... Ma solo dopo aver cominciato a scrivere i romanzi, e soprattutto quando $\mathrm{Il}$ Nome della rosa ha avuto grande successo in tutto il mondo grazie alle numerose traduzioni, Eco ha capito che tra l'autore e il lettore si pone un'altra inevitabile ed importante categoria - il traduttore. Fin da allora ha dedicato molte energie non solo ad opere di carattere teorico riguardanti la traduzione (il cui culmine è rappresentato da Dire quasi la stessa cosa), ma anche a una sorta di percorso formativo dei suoi traduttori modello. Questo rapporto originale, se non unico, in cui l'autore aiuta i propri traduttori e li consiglia, ha contribuito all'altissima qualità delle traduzioni, ma non solo.

"mirelarado@gmail.com

${ }^{1}$ U. Eco, Dire quasi la stessa cosa, Milano, Bompiani, 2010, p. 143. 
Ha accentuato il carattere plurale delle interpretazioni possibili dei romanzi o di passi tratti dai medesimi.

Ed è proprio il rapporto che nasce e si potenzia lungo quest'asse comunicativo, che ci consente di crescere professionalmente (e umanamente), imparando noi molto dal Professor Eco, e imparando lui a scoprire, attraverso le nostre domande, sfumature e soluzioni interpretative alle quali non aveva pensato in fase di scrittura, siccome le nostre lingue e le nostre culture spesso impongono diverse soluzioni, che neppure un autore di grande esperienza può prevedere.

Questo nostro contributo offre la possibilità di constatare che le soluzioni suggerite dallo scrittore non sono universali e non possono essere sempre applicate in tutte le lingue. Proprio per questa ragione, abbiamo scelto di cominciare con un esempio al quale Umberto Eco ha già fatto ricorso, anche se, abbiamo approfondito la sua analisi, arricchendola con nuovi esempi e con un esperimento fatto in classe. A pagina 151 del Dire quasi la stessa cosa Eco dice: «Sempre nel mio Pendolo di Foucault metto in bocca ai personaggi citazioni letterarie. La funzione di queste citazioni è di dimostrare l'incapacità di questi personaggi di guardare al mondo se non per interposta citazione». ${ }^{2}$

Seguono la citazione e le traduzioni in inglese, francese, tedesco, spagnolo e catalano:

Ma tra picco e picco si aprivano orizzonti interminati - al di là della siepe, come osservava Diotallevi verbalizzando giudiziosamente le nostre scoperte. ${ }^{3}$

Mais entre un pic et un autre s'ouvraient des horizons infinis-au-dessus des étangs, au-dessus des vallées, comme observait Diotallevi. ${ }^{4}$

At every curve the peaks grew, some crowned by little villages; we glimpsed endless vista. Like Darién, Diotallevi remarked. ${ }^{5}$

Doch zwischen den Gipfeln taten sich endlose Horizonte auf-jenseits des Heckenzaunes, wie Diotallevi bemerkte. ${ }^{6}$

${ }^{2}$ U. Eco, Dire quasi la stessa cosa, cit., p. 151.

${ }^{3}$ U. Eco, Il pendolo di Foucault, Milano, Bompiani, 1988, p. 204.

${ }^{4}$ U. Eco, Le pendule de Foucault, trad. di Jean-Noël Schifano, Paris, Grasset, 1990, p. 342 .

${ }^{5}$ U. Eco, Foucault's Pendulum, trad. di William Weaver, San Diego, A Helen and Kurt Wolff Book, 1989, p. 335.

${ }^{6}$ U. Eco, Das Foucaultsche Pendel, trad. di Burkhart Kroeber, München, Deutscher Taschenbuch Verlag, 1989, p. 393. 
Pero entre pico y pico se abrián horizontes illimitados: el sublime espacïoso llano, como observaba Diotallevi...?

Però entre pic i pic s'obrien horitzons interminables: tot era prop i lluny, i tot tenia com una resplendor d'eternitat, corn ho observava Diotallevi... ${ }^{8}$

Eco brevemente conclude che ciascun traduttore ha inserito un richiamo a un passo della propria letteratura riconoscibile dal lettore a cui la traduzione mirava e conclude che si sarebbe perso qualcosa se la citazione fosse stata tradotta alla lettera. Per verificare se questa osservazione funziona anche per altre lingue, abbiamo aggiunto anche la traduzione in russo, croato e serbo:

Дали между пиками казались неизмеримыми - прекрасное далеко, подытожил Диоталлеви. ${ }^{9}$

Ali između vrha i vrha otvarali su se beskonačni horizonti - s one strane ograde, kako je primećivao Diotalevi. ${ }^{10}$

Ali između vrhova otvarali su se beskrajni vidici - obzorja najkrajnjeg, kao što se izrazio Diotalevi. ${ }^{11}$

Abbiamo tentato di trovare da dove derivano le citazioni tradotte ed ecco che cosa siamo riusciti a trovare:

«Au-dessus des étangs, au-dessus des vallées», Baudelaire, Élévation (traduzione francese);

«Silent, upon a peak in Darien», Keats, On first looking into Chapman's Homer (traduzione inglese);

«que - del sublime espacioso llano, al huésped al caminar reduciendo», Gongora, Soledad primera (traduzione spagnola);

«Tot era prop i lluny, i tot tenia, Com una resplendor d'eternitat», Joan Maragall, Torno de la dolçor de les muntanyes (traduzione catalana).

${ }^{7}$ U. Eco, El pendulo de Foucault, trad. di Ricardo Pochtar, Buenos Aires, Editorial Lumen-Bompiani, 1989, p. 301.

${ }^{8}$ U. Eco, El pendol de Foucault, trad. di Antoni Vicens Barcelona, Destino, 1989, p. 323 .

9 У. Еко, Маятник Фуко, trad. di Елена Костюкович, Санкт-Петербург, Симпозиум, 2002, р. 380.

${ }^{10}$ U. Eco, Foucaultovo njihalo, trad. di Lia Paić, Zagreb, Izvori, 2003, p. 348.

${ }^{11}$ U. Eко, Fukoovo klatno, trad. di Mirela Radosavljević e Aleksandar Levi, Beograd, Narodna knjiga, 2001, p. 385. 
Non siamo riusciti a trovare da dove provengono le citazioni in tedesco e in russo, anche se un collega, docente di quest'ultima lingua, ci ha indicato che esiste una famosa canzone russa omonima. $\mathrm{Ci}$ pare che, per capire se le date citazioni siano adatte o no, dobbiamo prendere in considerazione anche questo fatto: il verso Al di là della siepe, tratto dalla poesia L'infinito di Giacomo Leopardi, è uno dei più celebri della letteratura italiana, quasi al livello di «nel mezzo del cammin di nostra vita», «chiare fresche e dolci acque», «l'albero a cui tendevi la pargoletta mano», «dolce e chiara è la notte e senza vento». Siamo sicuri che quasi ogni lettore italiano che si imbatte nel medesimo, riesce subito a recepire questo riferimento, soprattuttto perché si menziona la parola siepe. Se introducessimo un coefficiente di riconoscibilità come indicatore del livello di ricezione, stabilendo una scala da 1 a 100, potremmo dire che questa citazione ha il valore di 99. Ci pare che questo coefficiente sia molto alto, tanto alto che alcune rese, come quelle in francese e spagnolo, non abbiano saputo raggiungerlo.

Il traduttore catalano ha trovato una soluzione originale. Ha deciso di usare due versi e di rivelare al proprio lettore che si tratti di una citazione. Essendo la poesia catalana meno ricca di quella italiana, questo verso viene riconosciuto dai lettori catalani. Per quanto riguarda la soluzione di William Weaver, siamo sicuri che si sia trovato davanti ad un enorme ostacolo poiché è molto difficile trovare una soluzione che sia ugualmente efficace tanto per il lettore inglese, quanto per quello americano, per non parlare di quello australiano o addirittura africano. Il lettore americano sicuramente avrebbe gradito un verso di Poe o Walt Whitman. In ogni caso, la sua scelta ha un basso coefficiente di riconoscibilità, ma forse proprio questa era la sua intenzione.

In un primo momento anche noi avevamo pensato di seguire la stessa strada e di inserire un verso della nostra letteratura di pari riconoscibilità. $\mathrm{Ci}$ sono venuti in mente poeti romantici e famosi versi che sicuramente avrebbero potuto raggiungere un altissimo coefficiente di riconoscibilità. Purtroppo, abbiamo dovuto concludere che una soluzione simile non avrebbe funzionato per il lettore serbo, paradossalmente, proprio perché la tecnica dell'addomesticamento può funzionare solo nei casi di bassissimo coefficiente di riconoscibilità, almeno per paesi non abituati a declinare la propria cultura su vaste latitudini o a percepirla come universale. In effetti, il lettore serbo avrebbe riso se avesse scoperto in Diotallevi un fine conoscitore dei versi di Jakšić o Radičević, mentre nessuno si meraviglia quando Diotallevi cita Baudelaire, Keats o Gongora. Perciò abbiamo deciso di "salvare" Leopardi, cercando di non tradire l'intenzione autoriale. 
Per fortuna, L'infinito leopardiano è una delle più tradotte poesie in croato e in serbo. Tra le traduzioni di Alfirović, Miličić, Nazor, Mraović, Živković-Mandolfo, Smerdal e Machiedo, abbiamo scelto la traduzione del verso che ci è parsa la più poetica, quella di Simo Miličić - obzorja najkrajnja. Siamo consapevoli che il coefficiente di riconoscibilità sia prossimo allo 0, ma crediamo di essere riusciti a stuzzicare la curiosità del lettore serbo, e a dimostare che Diotallevi guarda il mondo attraverso gli occhiali della letteratura.

Anche dopo la pubblicazione della nostra traduzione non abbiamo smesso di occuparci di questo problema. Durante le lezioni di traduttologia abbiamo fatto un esperimento per verificare se i nostri studenti siano in grado di riconoscere il detto verso. ${ }^{12}$ Non abbiamo comunicato loro la soluzione proposta dall'autore, ma gli abbiamo chiesto di trovare un verso nella poesia serba che avrebbero inserito nella traduzione del brano. I risultati dell'esperimento sono stati davvero interessanti. Dobbiamo dire che tutti i nostri studenti hanno riconosciuto il passo di Leopardi e hanno trovato bellissimi versi nella nostra poesia:

«k’o glas u dubini noći», Jovan Dučić, Tama;

«ali su sunca na kraj sveta», Jovan Dučić, Suncokreti;

«da mi je da sam u predelima onim», Vladislav Petković Dis, Utoplljene duše;

«k’o da s' onog sveta dolazi», Đuro Jakšić, Na liparu;

«u blistavoj senci smaragdovih gora», Jovan Dučić, Zalazak sunca;

«i u mojoj duši produženo vidim ovo mirno more», Jovan Dučić, Podne;

«vasiona pukla pusta», Laza Kostić, Među zvezdama;

«i sjajne kapi sa bezbroj rubima rasipaju se dok tone jesenje sunce», Aleksa Šantić, Jedna suza;

«nejasno nebo nad nama se svodi», Jovan Dučić, De Profundis;

«onamo na vrhu, ko dan jedan beo», Aleksa Šantić, Senke;

«leži beskonačan vrh, zaspalih vala», Jovan Dučić, Pokraj vode;

«kao da se nebo bliže zemlji svija», Aleksa Šantić, Svijetli put.

${ }^{12}$ Occorre dire che questo progetto si è svolto grazie all'idea e sotto la vigilante cura della professoressa Mirka Zogović. 
Ciò che però ci ha colpito di più, sono le motivazioni che stanno dietro tali scelte. Di seguito riportiamo le più interessanti:

La siepe rappresenta un limite tra due spazi differenti e separati: da una parte c'è il buio e dall'altra la luce. (B. Č.)

Mi pare che questo verso contrasti volutamente con quello precedente e che Diotallevi provi emozioni molto forti nell'osservare il panorama. (J. J.)

Ho scelto di sostituire l'immagine di nuvole che vagano per l'infinito orizzonte blu all'immagine di picchi all'orizzionte che sembrano una siepe. (D. M.)

Il verso che ho scelto ha lo stesso ritmo, la stessa forma e sonorità del verso leopardiano. (Т. Đ.)

La mia scelta è legata al fatto che questo verso, come quello originale, accende nuove speranze e aumenta l'entusiasmo di colui che vede questa immagine. (M. S.)

La siepe rappresenta il confine tra la mente umana e l'inafferrabile, penso che questo verso trasmetta la stessa idea. (I. N.)

Mi sembra che il verso da me scelto esprima lo stesso concetto di fantasia e paura, ma ho cercato di trovarne uno che corrispondesse bene anche all'endecasillabo sciolto dell'originale. (M. P.)

Tutto ciò ci induce a trarre una conclusione che oltre alle idee dell'autore non si possa trascurare neanche l'intentio operis. Il pendolo di Foucault è ricco di spunti, di citazioni, di riferimenti, di interrogativi, di stimoli, di colpi di scena grazie all'erudizione e alla capacità di Umberto Eco di connettere i più diversi campi del sapere, ottenendo sempre ottimi risultati tanto in campo narrativo, quanto in ambito filosofico e teorico. Questo romanzo nasconde numerose interpretazioni e di conseguenza non esiste un modello unico, un punto di riferimento saldo che potrebbe soddisfare tutte le intenzioni. A volte le perdite e compensazioni sono inevitabili e il traduttore dei romanzi di Eco si trasforma in una specie di investigatore che senza indizi deve risolvere un omicidio complicato brancolando nel buio. In questo difficile compito i traduttori devono prendere in considerazione non solo il fatto che Umberto Eco non insiste sulla correttezza dei termini, ma sulla riproduzione dello stesso effetto, con i problemi specifici delle lingue in cui certi difficili brani vengono tradotti.

Vi è un altro esempio interessante di cui adesso vorremmo parlare. Sempre ne Il pendolo di Foucault, ricchissima fonte di citazioni letterarie, abbiamo trovato il seguente brano: 
Chi?

Ci hanno fatto credere che da una parte c'è la grande arte, quella che rappresenta personaggi tipici in circostranze tipiche, e dall'altra il romanzo d'appendice, che racconta di personaggi atipici in circostanze atipiche. Pensavo che un vero dandy non avrebbe mai fatto all'amore con Scarlet O'Hara e neppure con Costanza Bonacieux o con la Perla di Labuan... Le donne sono più simili a Milady che a Lucia Mondella. ${ }^{13}$

Ed eccole le traduzioni:

$-i$ Quiénes?

- Nos han hecho que por un lado está el gran arte, el que representa personajes típicos en situaciones típicas, y por el otro la novela por entregas, que habla de personajes atípicos en situaciones atípicas. Pensaba que un verdadero dandy nunca haría el amor con Scarlett O'Hara y ni siquiera con Costance Bonacieux o con la Perla de Labuan... Les mujeres se parecen más a Milady que a Madame Bovary. ${ }^{14}$

- Кто?

- Убеждали, будто существует, с одной стороны, высокое искусство, описывающее типические характеры в типических обстоятельствах, а с другой стороны роман-газета и там характеры нетипические и обстоятельства тоже. Я думал, что настоящие денди не ухаживают за Скарлетт О'Харами и за Консанциями Бонасье... И героинь Сальгари им даром не надо... Женщины похожи на маркизу ангелов Анжелику а не на ангельскую Беатриче. ${ }^{15}$

- How?

- Making us believe that on one hand there is Great Art, which portrays typical characters in typical situations, and on the other hand you have the thriller, the romance, which portrays atypical characters in atypical situations. No true dandy, I thought, would have made love to Scarlett O'Hara or even to Constance Bonacieux or Princess Daisy... Women are a lot more like Milady than they are like Little Nell...16

${ }^{13}$ U. Eco, Dire quasi la stessa cosa, Milano, Bompiani, 2010, pp. 523-524.

${ }^{14}$ U. Eco, El pendulo de Foucault, trad. di Ricardo Pochtar, Buenos Aires, Editorial Lumen-Bompiani, 1989, p. 447.

15 У. Эко, Маятник Фуко, trad. di Елена Костюкович, Санкт-Петербург, Симпозиум, 2002, р. 562.

${ }^{16}$ U. Eco, Foucault's Pendulum, trad. di William Weaver, San Diego, A Helen and Kurt Wolff Book, 1989, p. 495. 
- Qui?

- On nous a fait croire que d'un côté il y a le grand art, celui qui représente des personnages typiques dans des circonstances typiques, et de l'autre le romanfeuilleton qui racconte l'histoire des personnages atypiques dans des circonstances atypiques. Je pensais qu'un vrai dandy n'aurait jamais fait l'amour avec Scarlett O'Hara ni avec Constance Bonacieux ou Aurore de Caylus non plus... Les femmes ressemblent plus à Milady qu'à Clélia Conti. ${ }^{17}$

\begin{abstract}
"Wer?"
"Man hat uns eingeredet, auf der einen Seite wäre die Große Kunst, die Hochliteratur, die typische Personen in typischen Umständen darstellt, und auf der anderen die Trivialliteratur, die atypische Personen in atypischen Umständen darstellt. Ich glaubte, ein wahrer Dandy würde nie mit Scarlett O'Hara, nicht mal mit Constance Bonacieaux oder gar mit Angélique... Die Frauen sind Milady ähnlicher als Anna Karenina."18

- Кой?

- Накараха ни да повярваме, че от една страна съществува Голямото изкуство, това, което представя типични герои в типични обстоятелства, а от друга, романът подлистник, който разказва за нетипични герои в нетипични обстоятелства. Бях убеден, че един истински «денди» никога не би се любил нито със Скарлет О’Хара, нито с Констанс Бонасьо, нито дори с Лабуанската Перла на Салгари... Жените приличат повече на Миледи, отколкото на Мадам Бовари. ${ }^{19}$
\end{abstract}

«Tko?»

«Uvjerili su nas da s jedne strane postoji velika umjetnost, ona koja prikazuje tipične likove u tipičnim okolnostima, a s druge strane roman u nastavcima koji pripovijeda o atipičnim likovima u atipičnim događanjima. Mislio sam da pravi dandy nikada ne bi vodio ljubav sa Scarlett O’Hara, Costanzom Bonacieux ili s Perlom di Labuan. Žene su sličnije Milady nego Luciji Mondella». ${ }^{20}$

«Ko nas je prevario?»

«Oni koji su nas naučili da s jedne strane postoji velika književnost koja nam prikazuje tipične likove u tipičnim događajima, a s druge strane roman u nastavcima koji pripoveda o netipičnim likovima u netipičnim događajima. Mislio sam da pravi

${ }^{17}$ U. Eco, Le pendule de Foucault, trad. di Jean-Noël Schifano, Paris, Grasset, 1990, p. 501.

${ }^{18}$ U. Eco, Das Foucaultsche Pendel, trad. di Burkhart Kroeber, München, Deutscher Taschenbuch Verlag, 1989, p. 581.

${ }^{19}$ У. Еко, Махалото на Фуко, trad. di Бояна Петрова, София, Бард, 1992, p. 501.

${ }^{20}$ U. Eco, Foucaultovo njihalo, trad. di Lia Paić, Zagreb, Izvori, 2003, p. 510. 
džentlmen nikad ne bi mogao da vodi ljubav sa Skarlet O'Harom, pa ni Konstancom Bonasjo, ili Marijanom, Biserom Labuana... Žene više podsećaju na Miledi iz Dimina Tri musketara nego na Lučiju Mondelu iz Manconijevih Verenika».21

Dunque, Eco esprime il suo pensiero riguardo la Trivialliteratur, già esplicitato in altre opere teoriche, e subito dopo nomina alcuni personaggi femminili, appartenenti tanto alla grande letteratura quanto alla «paraletteratura». E se il brano di per sé non costituisce alcun problema per i traduttori, non tutti i personaggi sono "sopravvissuti" nelle diverse traduzioni. Scarlett O'Hara e Constance Bonacieux sono presenti in tutte le versioni qui citate, ma non la Perla di Labuan. In inglese diventa Princess Daisy, in francese Aurore de Caylus, in tedesco Angélique, nella traduzione bulgara si menziona anche Salgari, mentre la Костюкович propone una soluzione generica trasformando la Perla di Labuan in una delle "eroine" di Salgari. Noi abbiamo deciso di aggiungere il nome del personaggio Marianna. Tutti noi sappiamo bene che Salgari è una "passione" di Umberto Eco, ma siamo altrettanto sicuri che lui non insisterebbe perché il traduttore mantenesse questo personaggio a tutti i costi, soprattutto perché la fama di Salgari varia a seconda del paese. Ecco perché non dobbiamo meravigliarci che la Perla di Labuan sia rimasta nella traduzione spagnola dal momento che i romanzi di Salgari tutti tradotti godono di grande popolarità in Spagna come El Corsario Negro, La hija del corsario, El continente misterioso, El capitan del Djumna, Aquilas de la estepa, Los piratas de la Malasia, Los tigres de la Malasia, Sandokan, El leon de Damasco, Reina de los Caribes. La soluzione francese ci pare ideale. Al posto della Perla di Labuan, Schifano cita Aurore de Caylus. Il traduttore francese ha scelto questo personaggio del romanzo Le bossu di Paul Féval probabilmente perché il libro di questo scrittore francese racconta le avventure della figlia segreta del duca di Nevers che lasciano il lettore senza fiato, ma forse anche perché questo personaggio ha avuto popolarità anche sul grande schermo, come la Perla di Labuan. Burkhart Kroeber ha trovato un'ottima soluzione, salvando capra e cavoli. Angélique, di Serge e Anne Golon, l'eroina di 13 romanzi pubblicati nel periodo 1957-1985 risulta popolare in tutta l'Europa, ma è anche una "beniamina" di Eco, in quanto spesso citata nelle sue opere. Quella di Елена Костюкович ѐ una scelta chiara e cioè di lasciare al lettore russo la libertà di scegliere tra una delle numerose eroine salgariane quali Jolanda o Honorate Van Guld. La traduttrice bulgara ha aggiunto il nome dello scrittore per aiutare il lettore a riconoscere il personaggio, forse non tanto noto nel suo Paese, mentre noi abbiamo fatto ricorso ad una mossa

${ }^{21}$ U. Eко, Fukoovo klatno, trad. di Mirela Radosavljević e Aleksandar Levi, Beograd, Narodna knjiga, 2001, p. 562. 
scaltra, aggiungendo anche il vero nome del personaggio, Marianna, perché sotto questo nome è ancora oggi conosciuta in Serbia, come la donna amata da Sandokan nel famoso teleromanzo degli anni settanta con Kabir Bedi e Carol André (oltre a interpretare Lady Marianna Guillonk l'attrice francese ha interpretato anche la duchessina Van Guld ne Il corsaro nero). L'unica soluzione che ci pare sbagliata, o quantomeno contestabile, è quella del traduttore americano William Weaver. Princess Daisy, infatti, non può essere messa sullo stesso piano di altre eroine in quanto protagonista di un romanzo di Judith Kranz pubblicato nel 1978, dunque alcuni anni prima de Il pendolo di Foucault. A differenza di Angélique che ha lottato per essere riconosciuta, trovandosi nella scomoda posizione di essere molto amata dai lettori ma altrettanto osteggiata dai critici, Princess Daisy non ha ancora trovato il suo posto nella storia della letteratura e dubitiamo che mai lo troverà. Può darsi che questo riferimento sia stato usato come una specie di cripto-pubblicità per il mercato americano.

Ma le soluzioni proposte da quasi tutti i traduttori per la coppia MiladyLucia Mondella hanno soddisfatto solo una parte dell'intenzione autoriale, quella cioè di paragonare un personaggio femminile della paraletteratura ad un'eroina della grande letteratura, come a voler sottolineare che nella vita quotidiana è più facile incontrare persone simili ai personaggi cardine della paraletteratura. Ma pensiamo che vi sia un altro elemento importante e che Belbo non abbia scelto queste due donne a caso. Ci pare che Milady e Lucia rappresentino anche due modi antitetici e inconciliabili di essere donna, cioè due facce diverse della femminilità, quasi uno stereotipo della mora cattiva e della bionda angelica. L'infernale Milady non sceglie le armi per ottenere quello che vuole, mentre l'angelicata Lucia, uno dei personaggi più morali della storia della letteratura, accetta tutte le sciagure con diginità e umiltà. Ecco perché tutte le eroine proposte da vari traduttori quali Madame Bovary (spagnolo e bulgaro), Anna Karenina (spagnolo) come sostituzioni a Lucia non ci sembrano credibili. Little Nell, d'altro canto (tradotta come Lenuccia in italiano) di Dickens (The old curiosity shop) è secondo noi troppo giovane, poichè muore ancora ragazzina, ma tra i personaggi dickensiani ce n'è un altro, Little Dorrit, che sembra molto più adatto ad indossare i panni logori ed intrisi di sventura di Lucia. Clèlia Conti (La chartreuse de Parme) rappresenterebbe la soluzione migliore per diverse ragioni. Ė interessante la soluzione della traduttrice russa che ha optato per un altro tipo di opposizione, tra una donna virtuosa, ma carnale, di provenienza triviale e una donna simbolo della spiritualità e della castità. Noi abbiamo deciso di mantenere Lucia Mondella specificando che si tratti di un'eroina del Manzoni, strizzando l'occhio al nostro lettore. Resta il fatto che uno dei più importanti personaggi femminili della storia della letteratura 
italiana, non solo non abbia raggiunto la stessa fama in altri paesi, ma che sia addirittura quasi sconosciuta al grande pubblico, forse anche a causa delle pochissime traduzioni.

Aggiungiamo che dopo questo esempio vengono menzionati due personaggi maschili, Fu Manchu e Nathan il Saggio. Il personaggio di Sax Romer rimane in tutte le versioni, tranne in quella russa, nella quale viene sostituito dal Profesor Moriarty, ma è altresì molto interessante che in russo e in spagnolo Nathan diventi Pierre Besuchov.

Un altro esempio analogo lo fornisce il riferimento a Giorgio Baffo. Nel capitolo 9 de Il Pendolo di Foucault si menzionano i suoi sonetti: «Terzo manoscritto in una settimana, uno sul diritto bizantino, uno sul Finis Austriae e terzo sui sonetti del Baffo».

Giorgio Baffo (1694-1768), autore di vari sonetti impudici scritti in dialetto veneziano, può tranquillamente comparire anche nella traduzione poichè poco conosciuto anche in Italia, ma è la soluzione di Wiliam Weaver che ci pare ideale, avendo lui trovato uno scrittore simile a Baffo, John Willmot - Earl of Rochester. In questo caso la tecnica dell'addomesticamento funziona bene. Dicasi lo stesso per la soluzione spagnola, dove al posto di Baffo compare Aretino, un altro dei poeti lascivi.

L'intertestualità caratterizza tutte le opere di Eco, ma diventa dominante nel romanzo La misteriosa fiamma della regina Loana. ${ }^{22}$ Oltre alle citazioni vere e proprie, le cui fonti sono riportate nel libro, ci sono centinaia di riferimenti letterari, questa volta mescolati a proverbi, libretti, dialoghi dei film, discorsi politici, fumetti, canzoni popolari, filastrocche e tutto quello che riguarda il costume italiano tra gli anni trenta e cinquanta. Questa lunga lista avrebbe portato ogni traduttore sull'orlo della disperazione totale. Siamo dell'avviso che in questo romanzo la tecnica dell'addomesticamento e dell'adattamento debba essere usata, pur cercando di mantenere la principale intenzione autoriale che è quella di rievocare, presso il pubblico italiano, la memoria sbiadita di tale periodo. Citiamo una di queste "torri di Babele" delle pagine 22-23, premettendo che la medesima richieda un'attenta analisi. Malgrado una lunghissima ricerca, alcune citazioni sono rimaste un mistero anche per noi:

E infatti la mia testa non era vuota, vi vorticavano memorie non mie, la marchesa uscì alle cinque, nel mezzo del cammin di nostra vita, Ernesto Sabato e la donzelletta vien dalla campagna, Abramo generò Isacco Isacco generò Giacobbe, Giacobbe generò Giuda e Rocco i suoi fratelli, il campanile batte la mezzanotte santa e fu

${ }^{22}$ U. Eco, La misteriosa fiamma della regina Loana, Milano, Bompiani, 2014. 
allora che vidi il pendolo, sul ramo del lago di Como dormono gli uccelli dalle lunghe ali, monsieurs les anglais je me suis couché de bonne heure, qui si fa l'Italia o si uccide un uomo morto, tu quoque alea, soldato che scappa arrestati sei bello, fratelli d'Italia ancora uno sforzo, l'aratro che traccia il solco è buono per un'altra volta, l'Italia è fatta, ma non s'arrende, combatteremo all'ombra ed è subito sera, tre donne intorno al cor e senza vento, l'inconscia zagaglia barbara a cui tendevi la pargoletta mano, non chiedere la parola impazzita di luce, dall'Alpi alle Piramidi andò in guerra e mise l'elmo, fresche le mie parole nella sera pei quei quattro scherzucci da dozzina, sempre libera sull'ali dorate addio monti sorgenti dall'acque, ma il mio nome è Lucia, o Valentino, Valentino, storno, Guido io vorrei che al ciel si scoloraro, conobbi il tremolar l'arme gli amori, de la musique où marchent des colombes, fresca e chiara è la notte e il capitano, m'illumino pio bove, benché il parlar sia indarno li ho visti a Pontida, settembre andiamo dove fioriscono i limoni, qui comincia l'avventura del Pelide Achille, tintarella di luna dimmi che fai in principio la terra era siccome immobile, licht mehr licht über alles, contessa cos'è mai la vita? tre civette sul comò.

Questo è quanto siamo riusciti a scoprire, benchè molti punti interrogativi, rimasti insoluti, accompagnino i risultati di tale ricerca:

- «La marchesa uscì alle cinque» proviene da Paul Valéry: «La marquise sortit à cinq heures», ma la stessa frase è citata anche da L. Pirandello ne Il fu Mattia Pascal;

- «Nel mezzo del cammin di nostra vita», incipit della Divina Commedia;

- $\quad$ Ernesto Sabato, scrittore argentino;

- «La donzelletta vien dalla campagna», G. Leopardi, Sabato del Villaggio;

- «Abraamo generò Isacco; Isacco generò Giacobbe; Giacobbe generò Giuda», Vangelo secondo Matteo;

- Rocco e i suoi fratelli, film di Luchino Visconti;

- «Il campanile scocca [nel romanzo batte] la mezzanotte Santa», Guido Gozzano, La mezzanotte santa;

- $\quad$ « Fu allora che vidi il pendolo», incipit de Il pendolo di Foucault;

- «Sul ramo del lago di Como», incipit de I promessi sposi di A. Manzoni: «Quel ramo del lago di Como»);

- «Dormono gli uccelli dalle lunghe ali», poeta greco Alcmane, $I$ Frammenti, tradotto da numerosi traduttori e poeti italiani tra cui anche G. Pascoli e S. Quasimodo;

- «Monsieurs les anglais je me suis couché de bonne heure», M. Proust, À la recherche du Temps perdu; 
- «Qui si fa l'Italia o si muore», Giuseppe Garibaldi, battaglia di Calatafimi 1860, «O si uccide un uomo morto?»;

- $\quad$ «Tu quoque alea», Alea iacta est più Tu quoque, Brute, fili mi;

- «Soldato che scappa», prima parte del proverbio «Soldato che fugge, buono per un'altra volta»;

- «Arrestati sei bello», Mefistofele di Arrigo Boito: «Nè ancor dicesti all'attimo fuggente: Arrestati, sei bello!»;

- «Fratelli d'Italia», l'inno di Mameli;

- «Ancora uno sforzo», Marquise de Sade, Filosofia del budoir: «Forse francesi, ancora uno sforzo»;

- «L'aratro che traccia il solco è buono per un'altra volta», la prima parte proviene dal discorso di Mussolini: «È l'aratro che traccia il solco, ma è la spada che lo difende», mentre la seconda fa parte del proverbio sopra citato sul soldato che scappa;

- «L'Italia è fatta ma non si arrende», Camillo Cavour: «L'Italia è fatta. Tutto è salvo»; la seconda parte riprende la risposta del generale francese Cambronne ai generali inglesi durante la battaglia di Waterloo: «La guardia muore ma non si arrende»;

- «Combatteremo nell'ombra», traduzione di Erodoto: «Pugnabunt in umbra»;

- «Ed è subito sera» dalla omonima poesia di S. Quasimodo: «Ognuno sta solo sul cuor della terra / trafitto da un raggio di sole / ed è subito sera»;

- $\quad$ «Tre donne intorno al cor», Dante, Rime;

- «E senza vento», G. Leopardi, La sera del di di festa: «Dolce e chiara è la notte e senza vento»;

- «L'inconscia zagaglia barbara», Carducci, Odi Barbare, Per la morte di Napoleone Eugenio: «Questo la inconscia zagaglia barbara / prostrò, spegnendo li occhi di fulgida»;

- «A cui tendevi la pargoletta mano», Carducci, Pianto antico: «L'albero a cui tendevi la pargoletta mano»;

- «Non chiedere la parola», E. Montale: «Non chiederci la parola che mondi possa aprirti»;

- «Impazzita di luce», Eugenio Montale, Ossi di seppia: «Portami il girasole impazzito di luce»;

- «Dall'Alpi alle Piramidi», A. Manzoni, Il cinque Maggio: «Dall'Alpi alle Piramidi / dal Manzanarre al Reno»;

- «Andò in guerra e mise l'elmo», La partenza del crociato: «Passa un giorno, passa l'altro / Mai non torna il prode Anselmo / Perché egli era molto scaltro / Andò in guerra e mise l'elmo». Questa poesia scherzosa di Giovanni Visconti Venosta (Milano 1831-1906) 
fino agli anni cinquanta veniva stampata sul retro della copertina di molti quaderni di scuola ed era perciò conosciuta a memoria dalla maggior parte degli studenti italiani;

- «Fresche le mie parole nella sera», G. D’Annunzio, Alcyone - La sera fiesolana: «Fresche le mie parole ne la sera / ti sien come il fruscì che fan le foglie»;

- $\quad$ «Pei quei quattro scherzucci da dozzina», Giuseppe Giusti, Sant'Ambrogio: «Vostra eccellenza, che mi sta in cagnesco / per quei quattro scherzucci da dozzina»;

- «Sempra libera sulle ali dorati», G. Verdi, La Traviata: «Sempre libera degg'io / folleggiare di gioia in gioia» e Nabucco: «Va pensiero sulle ali dorate»;

- "Addio monti sorgenti dall'acque», A. Manzoni, I promessi sposi, capitolo VIII: «Addio, monti sorgenti dall'acque, ed elevati al cielo; cime inuguali, note a chi è cresciuto tra voi, e impresse nella sua mente, non meno che lo sia l'aspetto de' suoi più familiari»;

- «Ma il mio nome è Lucia», G. Puccini, La Bohème: «Sì, mi chiamo Mimì / ma il mio nome è Lucia»;

- «O Valentino Valentino storno», G. Pascoli, combinazione di Valentino e La cavalla storna;

- «Guido io vorrei», Dante, Rime: «Guido i' vorrei che tu e Lapo ed io»;

- «Che al ciel si scoloraro», F. Petrarca, Canzoniere, 3: «Era il giorno ch'al sol si scoloraro [nel romanzo «ciel» al posto di «sol»] / per la pietà del suo Fattore i rai»;

- «Conobbi il tremolar», Dante, Purgatorio, I, 117-20: «L'alba vinceva l'ora mattutina / che fuggia innanzi, sì che di lontano / conobbi il tremolar de la marina»;

- «L'arme gli amori», i versi iniziali di Orlando Furioso di Ariosto: «Le donne, i cavallier, l'arme, gli amori / le cortesie, l'audaci imprese io canto»;

- «De la musique où marchent des colombes», la prima parte forse di Paul Verlaine, «De la musique, avant toute chose», la seconda parte di Paul Valéry, Le cimetière marin: «Ce toit tranquille, où marchent des colombes / Entre les pins palpite, entre les tombes»;

- $\quad$ «Fresca e chiara è la notte», Leopardi, La sera del di di festa, ma Leopardi dice «dolce» al posto di «fresca»;

- «M'illumino», G. Ungaretti, Mattina: «M'illumino / d'immenso»;

- «Pio bove», G. Carducci, Il pio bove: «T'amo, o pio bove; e mite un sentimento»; 
- «Benché il parlar sia indarno», Petrarca, Italia mia, CXXVIII: «Italia mia, ben che 'l parlar sia indarno»;

- «Li ho visti a Pontida», Giovanni Berchet, Il giuramento di Pontida: «L'han giurato, li ho visti in Pontida / convenuti dal monte e dal piano»;

- «Settembre, andiamo dove fioriscono i limoni?», Settembre è una tra le più note poesie di D'Annunzio, dedicata all'attività dei pastori nel suo Abruzzo, mentre la seconda parte della frase è tratta da una poesia di Goethe, Mignon: «Conosci tu il paese dove fioriscono i limoni / nel verde fogliame splendono arance d'oro / un vento lieve spira dal cielo azzurro / tranquillo è il mirto e sereno l'alloro lo conosci tu bene»;

- «Qui comincia l'avventura del Pelide Achille», la traduzione di Monti dell'Iliade: «Cantami, o Diva, del Pelìde Achille l'ira funesta che infiniti addusse»;

- «Tintarella di luna», famosa canzone italiana «tintarella di luna / tintarella color latte», portata al successo da Mina;

- «Dimmi che fai», proverbio «Dimmi che fai e ti dirò chi sei»;

- «In principio la terra era», la Genesi: «In pricipio era il verbo» più «E Dio creò la terra»;

- «Siccome immobile», A. Manzoni, Il 5 maggio: «Ei fu siccome immobile»;

- «Licht mehr licht über alles», combinazione di Goethe e l'inno tedesco Deutschland, Deutschland über alles;

- «Contessa cos'è mai la vita?», G. Carducci, Jaufré Rudel: «Contessa, che è mai la vita? E' l'ombra d'un sogno fuggente»;

- «Tre civette sul comò», filastrocca: «Ambarabà Cicci Coccò / tre civette sul comò».

Ciascuno dei molti collage (il seguente arriva solo due pagine dopo) pone ogni traduttore davanti a un serio problema. Pensiamo che non ci sia un modello per tradurre questo tipo di testo perché abbiamo l'impressione che siffatti paragrafi provochino diverse "scosse" nel lettore. Il loro numero dipende dalle competenze del lettore, o dalla sua cultura, dalla sua età, ma anche dalla sua capacità di fare associazioni e di risolvere giochi enigmistici. Ecco perché anche noi abbiamo fatto ricorso ad un complicato miscuglio di traduzioni dei versi citati (come l'incipit della Divina Commedia, Tre donne intorno al cor, o Il 5 maggio di Manzoni, tradotto dal grande poeta croato Ivan Mažuranić). Il secondo metodo è quello di sostituire ai versi italiani meno conosciuti nel nostro Paese, quelli un po' più conosciuti. Così nella nostra versione compare il Petrarca di Voi ch'ascoltate in rime sparse il suono e il Cecco Angiolieri di Se fossi fuoco. Il terzo metodo è 
consistito nell'inserire celebri versi di vari scrittori stranieri (Goethe, Heine, Shakespeare, Pushkin, Baudelaire, Pablo Neruda, Emily Dickinson, Ana Ahmatova, E. A. Poe), alcuni dei quali ben noti grazie alle buone traduzioni prevalentemente fatte dai nostri poeti, come per esempio Annabel Lee. Le frasi in latino e francese sono rimaste intatte, anzi abbiamo ritradotto Erodoto in latino. I proverbi e la filastrocca sono addomesticati. Questo metodo, che di solito non funziona bene per i nostri lettori, in questo romanzo invece si è rivelato molto utile, addirittura essenziale. Così per tutte le descrizioni della nebbia abbiamo usato i versi della nostra poesia, ma anche alcuni passi della prosa. Poichè questo romanzo ha lo scopo di (ri)accendere la memoria dei lettori, abbiamo cercato di stimolare anche la mente dei nostri, di quelli di lingua serba. Ecco perché abbiamo deciso di tradurre la combinazione dei versi di impegno civile di Petrarca e di Berchet con due versi famosi della poesia patriottica serba di Aleksa Šantić e Đura Jakšić. Traducendo questo brano ci siamo accorti di alcuni piccoli errori nelle citazioni. Supponiamo che Eco li abbia commessi volutamente quasi a volerli attribuire alla memoria di un uomo appena svegliato dal coma, e quindi debole e fallace per ovvie ragioni. Ma d'altra parte, nessuno è perfetto e qualcosa può sfuggire anche all'attentissimo professor Eco. Sarebbe interessante sapere che cosa abbia pensato della traduzione degli errori. Ne Il Pendolo di Foucaualt ne abbiamo trovato uno, per quanto piccolo e veniale. Dopo le affermazioni di Belbo, convinto del fatto che tutta l'opera di Verne rappresenti una rivelazione iniziatica dei misteri del sottosuolo, Casaubon agginuge: «Come si chiama il protagonista delle Indie Nere? John Garral, quasi un anagramma di Graal» (capitolo 84). La nostra traduzione non ha completamente corretto l'errore di Eco o di Casaubon, ma abbiamo scelto deliberatamente di ridimensionare la sicumera di Casaubon: «Mi ricordo che il protagonista di uno dei romanzi di Verne, se non sbaglio proprio di Indie Nere [citato prima], si chiamava Garral, un anagramma di Graal».

Se non incorriamo in un errore, John Garral non esiste. Esiste invece un Joam Garral, protagonista del romanzo La Jangada, mentre nelle Indie nere i protagonisti sono James Starr e Simon Ford. In questo caso, abbiamo deciso di non correggere l'errore dell'autore, qualora non si tratti di una precisa scelta stilistica, ma di ridurne la nitidezza, eliminando il nome «Joan», aggiungendo un'ombra di dubbio che nell'originale non compare. 
Mirela Radosavljević - Aleksandar Levi

\title{
ZNAČAJ CITATA U ROMANU FUKOOVO KLATNO UMBERTA EKA I NJIHOVO PREVOĐENJE
}

\author{
(Rezime)
}

Pozivajući se na stavove samog U. Eka glede značaja citata u okviru nekog dela za njegovu višeznačnost i "otvorenost" u interpretaciji, autori, i sami prevodioci, usredsređuju se na nekolike primere Eku dragih citata u romanu Fukoovo klatno i analiziraju moguće interpretacije - zahvaljujući eksperimentu sa studentima-, kao i one u prevodima na razne jezike već prevodilačkim rešenjem utvrđene. 